\title{
Examining Unlock Journaling with Diaries and Reminders for In Situ Self-Report in Health and Wellness
}

\author{
Xiaoyi Zhang ${ }^{1}$, Laura R. Pina ${ }^{1,2}$, James Fogarty ${ }^{1}$ \\ ${ }^{1}$ Computer Science \& Engineering, ${ }^{2}$ Human Centered Design \& Engineering \\ DUB Group, University of Washington \\ \{xiaoyiz, lpina, jfogarty \}@cs.washington.edu
}

\begin{abstract}
In situ self-report is widely used in human-computer interaction, ubiquitous computing, and for assessment and intervention in health and wellness. Unfortunately, it remains limited by high burdens. We examine unlock journaling as an alternative. Specifically, we build upon recent work to introduce single-slide unlock journaling gestures appropriate for health and wellness measures. We then present the first field study comparing unlock journaling with traditional diaries and notification-based reminders in self-report of health and wellness measures. We find unlock journaling is less intrusive than reminders, dramatically improves frequency of journaling, and can provide equal or better timeliness. Where appropriate to broader design needs, unlock journaling is thus an overall promising method for in situ self-report.
\end{abstract}

\section{Author Keywords}

Personal informatics; self-tracking; experience sampling.

\section{ACM Classification Keywords}

H.5.2. Information interfaces and presentation (e.g., HCI).

\section{INTRODUCTION AND MOTIVATION}

The use of mobile devices for prompted in situ self-report has become common across a variety of disciplines, sometimes called experience sampling [3,15,23], diaries [8,34,36,41], or ecological momentary assessment $[21,40,44]$. Applications of these methods are too broad to enumerate, but research in the CHI community has included studies of mobile device adoption [34], of time management and interruptions $[17,24,26,35,38]$, of relating sensed behavior traces to explicit reports [20,27], of location privacy preferences [13], and informing and evaluating mobile and ubiquitous computing applications [7,11,12,14,41]. The CHI community is also actively exploring self-tracking through the lenses of personal informatics [32], the Quantified Self [10], and lived informatics $[16,18,37]$. We are particularly interested in the increasing applications of these techniques to self-monitoring in health and wellness (e.g., [6,28]).

Permission to make digital or hard copies of all or part of this work for personal or classroom use is granted without fee provided that copies are not made or distributed for profit or commercial advantage and that copies bear this notice and the full citation on the first page. Copyrights for components of this work owned by others than ACM must be honored. Abstracting with credit is permitted. To copy otherwise, or republish, to post on servers or to redistribute to lists, requires prior specific permission and/or a fee. Request permissions from Permissions@acm.org.

CHI'16, May 07-12, 2016, San Jose, CA, USA

Copyright is held by the owner/author(s). Publication rights licensed to ACM. ACM 978-1-4503-3362-7/16/05...\$15.00

DOI: http://dx.doi.org/10.1145/2858036.2858360
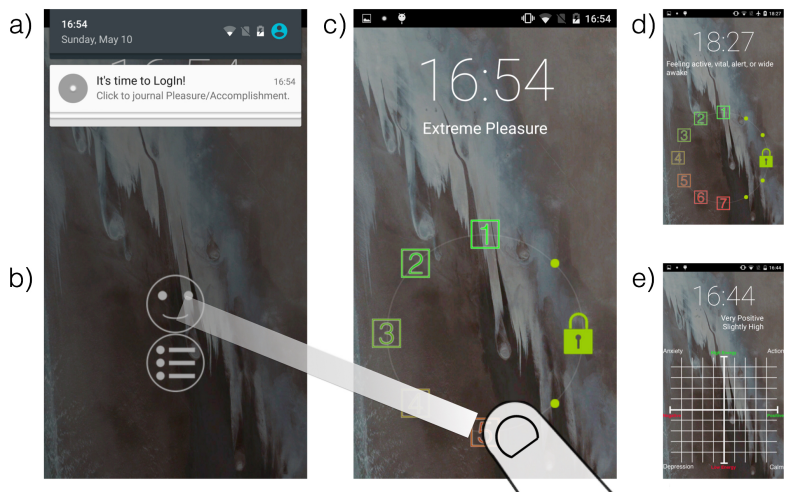

Figure 1. We examine unlock journaling, in which unlocking a phone also journals an in situ self-report. We present the first study comparing to diaries and notification-reminders.

Unfortunately, potential benefits of these techniques remain limited by their high burdens. Quantified Selfers report the burdens of self-tracking often create a fatigue that leads them to abandon self-tracking [10]. Experience sampling research has found the frequency of prompts must be limited to mitigate annoyance they create [19,27]. Intrusiveness of prompts can also be reduced using context awareness and decision theory methods to focus prompts at appropriate times and reduce the total number of prompts $[25,26,38]$. Health and wellness applications face the additional challenge that the act of journaling is often itself intended to promote mindfulness and self-monitoring (i.e., self-report serves both assessment and treatment [29]). Too much of a focus on sensing, automating data collection, or minimizing journaling may therefore undermine the entire purpose [42].

Initial methods used beepers (i.e., pagers) to prompt people to complete paper diaries [15]. This same basic approach continues in mobile interfaces that present a survey with multiple questions requiring minutes to complete. In contrast, recent work explores more lightweight journaling interfaces. Choe et al.'s SleepTight uses a phone lockscreen widget for journaling of potential sleep disruptors with as little as a single tap [9]. We build upon work by Truong et al., repurposing the otherwise wasted action of unlocking a phone (i.e., the slide to unlock gesture) to allow a small optional action [46]. Figure 1 illustrates our LogIn system, which modifies the unlock gesture to enable single-slide unlock journaling of health and wellness self-reports for sleepiness [22], pleasure and accomplishment [31], or mood [39].

Although repurposing the unlock gesture is appealing in its potential to reduce burdens of journaling, no prior work has 
compared this with traditional approaches. Researchers and designers thus lack information regarding how unlock journaling for health and wellness might be expected to perform in practice. We therefore present a field study in which 24 people each journal a health and wellness measure for 18 days. We compare unlock journaling with diaries and notification-based reminders, finding that unlock journaling is less intrusive than reminders, dramatically improves frequency of journaling, and can provide equal or better timeliness. Where appropriate to broader design needs, unlock journaling is thus a promising method for in situ self-report.

The specific contributions of this work therefore include:

- We expand the design of single-slide unlock journaling gestures to support (1) selection among multiple measures to journal, and (2) journaling a two-dimensional measure.

- We present the first field study comparing unlock journaling with traditional diaries and notification-based reminders for self-report of health and wellness measures.

\section{RELATED WORK}

The CHI community adopted diary studies as an alternative to field observation, which requires a researcher be present [36]. People often forget to journal, so methods evolved to provide reminders via beepers (i.e., pagers) [15], mobile devices [3], and text messages [1]. As devices became more common and capable, techniques have been updated to leverage advances in mobile media capture [8,34], automatic sensing [19,25], mobile notifications [5], and context awareness [25,26,38]. Enabled by these advances, an important thread of research has examined how those advances impact in-situ self-report $[3,5,8,43]$.We similarly contribute by examining the potential of unlock journaling as a new low-burden approach to in situ self-report, focusing on health and wellness applications.

We build directly on Truong et al.'s Slide to X technique [46]. This technique assigns a dual purpose to the unlock gesture, aiming to preserve its extreme simplicity while having that same gesture perform a simple, optional task. They propose Slide to QuantifySelf, but do not field such an application, nor do they compare to data collection with traditional diaries or notification-based reminders. We extend the single-slide gestures illustrated by Truong et al. to support common health and wellness measures. We also present the first field study comparing single-slide unlock journaling to traditional approaches. Additional examples of lightweight lockscreen interaction include small crowdsourcing tasks in Twitch [47], a crossing-based interface for email review in ProactiveTasks [2], and single-tap journaling via a lockscreen widget in SleepTight [9]. None of these provide the guidance we contribute on how single-slide unlock journaling might perform for a variety of health and wellness measures.

\section{DESIGN OF LOGIN}

LogIn is our implementation of unlock journaling, focused on validated health and wellness measures selected to be representative of needs in this space $[22,31,39]$. This section details our design of singe-slide unlock journaling in LogIn.
Our first measure is the Stanford Sleepiness Scale, a 7-point one-dimensional scale administered multiple times per day to identify sleep debt and patterns of alertness [22]. Such a scale is supported by the original Likert-type design in Slide to $X$ [46], though we modify the design to preserve Android conventions and avoid thumb occlusion. Drop targets appear when a person touches the unlock handle, a text explanation of a target is provided by sliding the handle to it, and releasing the handle then simultaneously journals the selection and unlocks the phone (Figure 1d, 2a).

Our second measure is for pleasure and accomplishment, implemented as a pair of 5-point one-dimensional scales. A person working with a therapist to assess and treat depressive symptoms may journal whether activities create a sense of pleasure or accomplishment, as such activities are important to behavioral activation in managing symptoms $[4,31,33]$. The original Slide to $\mathrm{X}$ interactions do not support single-slide selection of what to journal, so we introduce choosing from multiple handles to slide. A person chooses which scale to journal by touching the appropriate handle, then the interaction proceeds as before (Figure 1b, 1c, 2b).

Our third measure is Russell's Affect Grid, a two-dimensional measure of mood (i.e., pleasure and arousal) appropriate for journaling throughout the day [39]. The original Slide to X interactions do not support single-slide gesturing to journal in a two-dimensional scale. This is challenging in part because of the need to allow selection from the grid while preserving the default slide to unlock interaction (i.e., Figure 2d). We resolve this by introducing layers in the slide interaction. A person slides the handle to a grid icon, which activates the grid layer, then slides to the desired value and releases (Figure 1e, 2c), simultaneously journaling and unlocking.

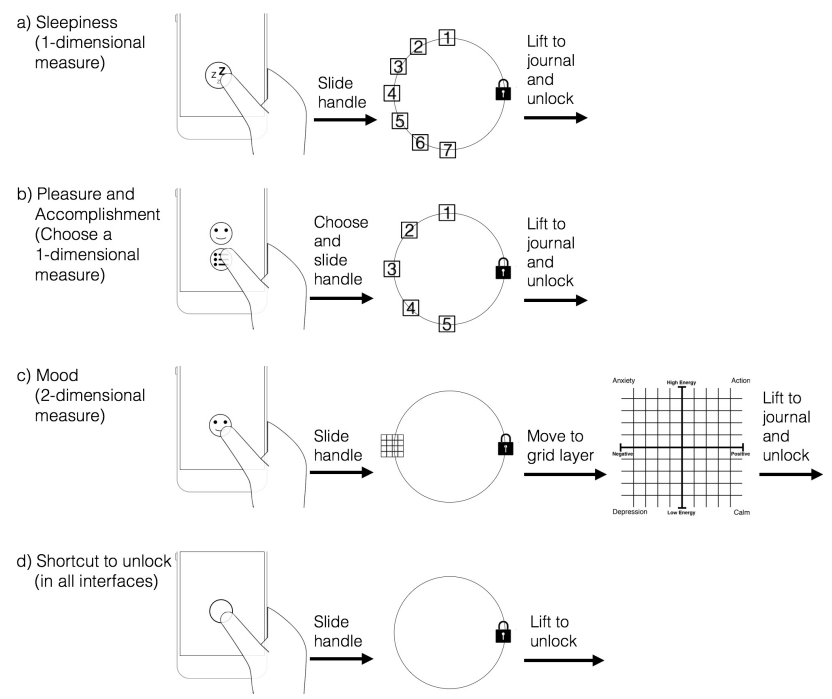

Figure 2. We design single-slide unlock gestures to support journaling of sleepiness (i.e., a rating scale), pleasure and accomplishment (i.e., choosing the scale to journal), and mood (i.e., a two-dimensional scale). All designs make journaling optional, preserving the simplicity of the default unlock. 
Journaling is always optional, and all designs preserve the default unlock gesture (Figure 2d). Journaling is therefore visible and easy but not required, as required journaling could result in people recording less reliable data while attempting to quickly access their phone. Although it is important our field evaluation is based in commonly-used measures, we also again note these measures were selected to be representative of needs in this space. Other health and wellness measures could likely be supported through these interactions or through designs based on their insights (e.g., choosing among layers via multiple slide targets).

Implementation. LogIn is implemented for Android, which provides a public API for customizing the lockscreen. Unlock journaling thus replaces the default unlock gesture (i.e., is not an additional step in the interaction). Any system password is preserved and entered after the unlock gesture (i.e., at the same point in the overall interaction sequence).

\section{FIELD EVALUATION}

Our evaluation examines unlock journaling in a field study focused on health and wellness self-report, comparing to traditional diaries and notification-based reminders.

\section{Design and Procedure}

We use a $2 \times 3$ within-subjects design, examining the impact of interfaces with unlock journaling (with, without) and notifications (none, traditional, aggressive) on intrusiveness, frequency, and timeliness of journaling in health and wellness.

Traditional notifications conditions generated a platform notification reminding the participant to journal (Figure 1a). Aggressive notifications added sound/vibration. Notifications were generated every 30 minutes in a configurable 12-hour window, applying a small random jitter to avoid grouping with any notifications from other applications at the same approximate time. The participant configured this window at the beginning of the study. For comparison, unlock journaling was only presented during this same window.

Base Functionality. All conditions provided an app which allowed journaling at will (i.e., participants could journal at any time, without needing to wait for a notification or first lock their phone). A simple app also visualized a participant's data, allowing them to view their journal at will. Note that the condition without unlock journaling and none notifications is a traditional diary (i.e., can enter and view data, but does not otherwise surface itself or remind participants to journal).

Journaling Domain. To maximize participant interest in the task, we screened participants for interest and allowed them to choose their journaling domain from sleepiness, pleasure and accomplishment, or mood. Each participant used different interfaces for journaling in their chosen domain over the course of their participation.

Procedure. Participants used their own Android phones to journal with LogIn over the course of 18 days. Each of the 6 conditions was used for 2 consecutive days, with a rest day between conditions. The LogIn app automatically switched interface conditions at midnight. To account for possible carryover effects (e.g., fatigue, learning, varying interest), we counterbalanced conditions using a balanced Latin Square.

Participation began with a brief interview, during which we installed LogIn on the participant's phone. We explained the study, then they chose their domain and configured their 12-hour journaling window. We emphasized compensation was not dependent on frequency of interaction, only a need to leave LogIn installed and return for a brief exit interview. Participants were compensated with a \$20 gift card.

\section{Participants}

We recruited through local email lists, linking to a questionnaire screening for use of a phone running Android 4.2 or above and for interest in three domains. From the eligible pool, we randomly selected 24 participants, reporting an average age of 26.1 years $(\min 19$, max 47 , med 24$)$, 14 male, 10 female. 11 chose to journal sleepiness, 6 pleasure and accomplishment, and 7 mood. Reporting motivation to journal in their chosen domain on a 7-point Likert scale, 15 reported motivation 7, 7 motivation 6 , and 2 motivation 5 .

\section{Results}

Participants journaled an average of 147 entries in 12 days (min 36, max 255, med 149), or 12.2 per day. When unlock journaling was active in the interface condition, they unlocked without journaling an average of 228 times, or 38 per day. We analyze their reported intrusiveness of conditions and their frequency and timeliness of journaling by condition.

We use mixed-model analyses of variance, with participant as a random effect (i.e., accounting for individual differences). We model domain and study day, but find no significant results (i.e., no differences by journaling domain and no carryover effects). We model unlock journaling, notifications, and their interaction. Figure 3 presents our primary results. To control for other independent variables, means reported in Figure 3 and this section are all least squares means.

Intrusiveness. At the end of each 12-hour study window, an alert asked participants to rate intrusiveness of that day's condition on a 5-point Likert scale. We find the addition of unlock journaling is reported as more intrusive ( 2.12 vs. 2.38 , $\left.F_{1,258}=11.7, p \approx .001\right)$. We find a main effect for notifications $\left(F_{2,258}=12.7, p<.001\right)$, and Tukey's HSD finds aggressive (2.94) is reported as more intrusive than traditional (2.27, $\left.F_{1,258}=51.3, p<.001\right)$, with both more intrusive than none $\left(1.55, F_{1,258}=218, p<.001, F_{1,258}=57.9, p<.001\right)$.

Of particular interest is a contrast between unlock journaling and notifications (e.g., a designer choosing one or the other to enhance a diary that has neither). Tukey's HSD finds addition of notifications is more intrusive than unlock journaling (2.22 vs. $\left.1.77, F_{2,258}=11.8, p \approx .001\right)$. Part of this may be that unlock journaling is surfaced during normal interaction, which P18 explained as: "I have so many different reminder and buzzing things and don't know which one is the journal reminder. But if I see this lockscreen, I know I am supposed to do it ... it is very easy." 
Frequency. We define frequency as the number of entries a person journals in a day. We find the addition of unlock journaling doubles frequency (16.1 vs. 7.9 entries per day, $\left.F_{1,258}=188, p<.001\right)$. We find a main effect for notifications $\left(F_{2,258}=31.4, p<.001\right)$. Tukey's HSD finds aggressive (14.3) and traditional (13.0) yield greater frequency than none $\left(8.8, F_{1,258}=57.5, p<.001, F_{1,258}=33.6, p<.001\right)$, but not significantly different from each other $\left(F_{1,258}=3.19\right.$, $p \approx .075$ ). Part of unlock journaling's higher frequency and a lack of improvement with aggressive notifications may be that notifications become less effective if delivered too often or too aggressively, contributing to intrusiveness instead of increasing frequency, as P7 explained: "for the days there were many notifications, I feel like 'Ugh, leave me alone".

We find a significant interaction between unlock journaling and notifications $\left(F_{2,258}=13.1, p<.001\right)$. Tukey's HSD finds the diary condition (i.e., none without unlock) yields significantly less frequency than other conditions (2.5, all pairs $p<.001)$. The diary condition provided no reminder to journal, and some reported forgetting entirely (e.g., P9 said "I rarely remember if I don't have any notifications and (the lockscreen) is shown nothing. You can see from what I did ... I probably hardly journal anything." Of 13 days that participants had a frequency of 0,10 are in the diary condition (including 1 from $\mathrm{p} 1$, who did not journal on 3 days and made only 36 entries over the entire study).

The contrast between unlock journaling and notifications is again of particular interest, and we find that unlock journaling improves frequency significantly more than traditional notifications (15.0 vs. 9.8, $\left.F_{1,258}=25.6, p<.001\right)$ or aggressive notifications (15.0 vs. $11.5, F_{1,258}=11.5, p \approx .001$ ).

Timeliness. Our final analysis examines timeliness separately from frequency, as some domains may require journaling near a particular time. Considering only cases where a self-report was provided, we examine average proximity to the nearest 30-minute interval (i.e., when notification was or would have been made). We define proximity as two-sided, ranging from 0 to 15 . We exclude 13 days with frequency 0 .

We find the addition of unlock journaling improves timeliness (7.8 vs. 9.3, $\left.F_{1,245}=49.5, p<.001\right)$. We find a main effect for notifications $\left(F_{2,245}=42.9, p<.001\right)$, and Tukey's HSD finds aggressive (7.3) improves timeliness over traditional $\left(8.8, F_{1,245}=33.8, p<.001\right)$, and both improve over none $\left(9.6, F_{1,245}=83.1, p<.001, F_{1,245}=11.9, p<.001\right)$.

We are again particularly interested in a contrast between unlock journaling and notifications (e.g., a designer choosing one or the other to enhance a diary that has neither). Tukey's HSD finds addition of unlock journaling or notifications to a diary to be indistinguishable in their impact on timeliness ( 8.6 vs. 9.3, $F_{2,245}=4.28, p \approx .040$, but failing the test's adjustment for multiple comparisons).

\section{DISCUSSION}

If choosing between unlock journaling or notifications, our results suggest unlock journaling will be perceived as less

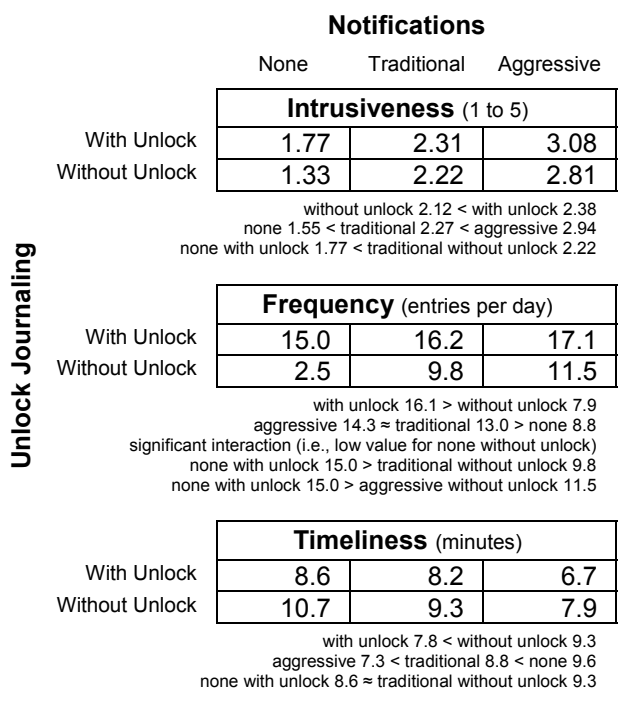

Figure 3. Summary of analyses, all as least squares means. Compared to notifications, unlock journaling is less intrusive, dramatically improves frequency, with equal or better timeliness.

intrusive (1.77 vs. 2.22), yield greater frequency of journaling (15.0 vs. 9.8), with comparable timeliness (8.6 vs. 9.3). Unlock journaling can also be combined with notifications, improving frequency and timeliness with a small increase in intrusiveness. These results motivate additional longer-term comparisons and studies of other characteristics of unlock journaling (e.g., different reporting windows, long-term fatigue, reliability of self-report collected in this manner).

Unlock journaling is limited to self-report that can be reduced to single-swipe gestures. We have extended the space of such gestures, but interesting opportunities for future work include pairing unlock journaling with other methods (e.g., with wearable eating detection [45] for lightweight verification of sensed data). LogIn also adds some complexity to a phone's unlocking mechanism. Although not a concern for our participants, this complexity could be a challenge for some.

Finally, Lathia et al. note the design of experience sampling implies bias in the resulting data [30]. This is unavoidable, as every method has a bias, but should be considered in applying a method. Experience sampling is already biased toward times a person can respond, and unlock journaling may magnify this to times when they are already accessing their phone. Depending on the application, this is potentially offset by the greater frequency of journaling.

There remain significant opportunities to extend this first field study comparing unlock journaling with traditional diaries and notification-based reminders in self-report of health and wellness measures. But our overall results suggest that, where appropriate to broader design needs, unlock journaling is a promising method for in situ self-report.

\section{ACKNOWLEDGMENTS}

This work is sponsored in part by the Intel Science and Technology Center for Pervasive Computing, Nokia Research, the AHRQ under award 1R21HS023654, and the NSF under awards OAI-1028195 and SCH-1344613. 


\section{REFERENCES}

1. Jacob Anhøj and Claus Møldrup. (2004). Feasibility of Collecting Diary Data from Asthma Patients through Mobile Phones and SMS (Short Message Service): Response Rate Analysis and Focus Group Evaluation from a Pilot Study. Journal of Medical Internet Research, 6(4), e42. http://doi.org/dfvrrg

2. Nikola Banovic, Christina Brant, Jennifer Mankoff, and Anind K. Dey. (2014). ProactiveTasks: The Short of Mobile Device Use Sessions. Proceedings of the International Conference on Human-Computer Interaction with Mobile Devices \& Services (MobileHCI 2014), 243-252.

http://doi.org/bbn9

3. Lisa Feldman Barrett and Daniel J. Barrett. (2001). An Introduction to Computerized Experience Sampling in Psychology. Social Science Computer Review, 19(2), 175-185. http://doi.org/d4tqhe

4. J.S. Beck and A.T. Beck. (2011). Cognitive Behavior Therapy: Basics and Beyond. Guilford Press.

5. Frank Bentley and Konrad Tollmar. (2013). The Power of Mobile Notifications to Increase Wellbeing Logging Behavior. Proceedings of the SIGCHI Conference on Human Factors in Computing Systems (CHI 2013), 1095-1098. http://doi.org/f2zs59

6. Lora E. Burke, Jing Wang, and Mary Ann Sevick. (2011). Self-Monitoring in Weight Loss: A Systematic Review of the Literature. Journal of the American Dietetic Association, 111(1), 92-102. http://doi.org/ct2ph2

7. Scott Carter, Jennifer Mankoff, and Jeffrey Heer. (2007). Momento: Support for Situated Ubicomp Experimentation. Proceedings of the SIGCHI Conference on Human Factors in Computing Systems (CHI 2007), 125-134.

http://doi.org/d69b3v

8. Scott Carter and Jennifer Mankoff. (2005). When Participants do the Capturing: The Role of Media in Diary Studies. Proceedings of the SIGCHI Conference on Human Factors in Computing Systems CHI (2005), 899-908.

http://doi.org/dqfsph

9. Eun Kyoung Choe, Bongshin Lee, Matthew Kay, Wanda Pratt, and Julie A. Kientz. (2015). SleepTight: Low-Burden, Self-Monitoring Technology for Capturing and Reflecting on Sleep Behaviors. Proceedings of the ACM International Joint Conference on Pervasive and Ubiquitous Computing (UbiComp 2015), 121-132.

http://doi.org/bbpb
10. Eun Kyoung Choe, Nicole B. Lee, Bongshin Lee, Wanda Pratt, and Julie A. Kientz. (2014). Understanding Quantified-Selfers' Practices in Collecting and Exploring Personal Data. Proceedings of the ACM Conference on Human Factors in Computing Systems (CHI 2014), 1143-1152. http://doi.org/bbpd

11. Karen Church, Mauro Cherubini, and Nuria Oliver. (2014). A Large-Scale Study of Daily Information Needs Captured In Situ. ACM Transactions on Computer-Human Interaction, 21(2). http://doi.org/bbpf

12. Sunny Consolvo, Predrag Klasnja, David W. McDonald, Daniel Avrahami, Jon E. Froehlich, Louis LeGrand, Ryan Libby, Keith Mosher, and James A. Landay. (2008). Flowers or a Robot Army?: Encouraging Awareness \& Activity with Personal, Mobile Displays. Proceedings of the International Conference on Ubiquitous Computing (UbiComp 2008), 54-63. http://doi.org/dk9hqb

13. Sunny Consolvo, Ian E. Smith, Tara Matthews, Anthony LaMarca, Jason Tabert, and Pauline Powledge. (2005). Location Disclosure to Social Relations: Why, When, \& What People Want to Share. Proceedings of the SIGCHI Conference on Human Factors in Computing Systems (CHI 2005), 81-90. http://doi.org/dk5d5v

14. Sunny Consolvo and Miriam Walker. (2003). Using the Experience Sampling Method to Evaluate Ubicomp Applications. IEEE Pervasive Computing, 2(2), 24-31. http://doi.org/cmbg6h

15. Mihaly Csikszentmihalyi and Reed Larson. (1987). Validity and Reliability of the Experience-Sampling Method. The Journal of Nervous and Mental Disease, 175(9), 526-536.

http://doi.org/bwb7km

16. Daniel A. Epstein, An Ping, James Fogarty, and Sean A. Munson. (2015). A Lived Informatics Model of Personal Informatics. Proceedings of the ACM International Joint Conference on Pervasive and Ubiquitous Computing (UbiComp 2015), 731-742. http://doi.acm.org/10.1145/2750858.2804250

17. James Fogarty, Scott E. Hudson, Christopher G. Atkeson, Daniel Avrahami, Jodi Forlizzi, Sara Kiesler, Johnny C. Lee, and Jie Yang. (2005). Predicting Human Interruptibility with Sensors. ACM Transactions on Computer-Human Interaction, 12(1), 119-146. http://doi.org/fpqwt3 
18. Thomas Fritz, Elaine M. Huang, Gail C. Murphy, and Thomas Zimmermann. (2014). Persuasive Technology in the Real World: A Study of Long-Term Use of Activity Sensing Devices for Fitness. Proceedings of the ACM Conference on Human Factors in Computing Systems (CHI 2014), 487-496.

http://doi.org/bbdv

19. Jon E. Froehlich, Mike Y. Chen, Sunny Consolvo, Beverly L. Harrison, and James A. Landay. (2007). MyExperience: A System for In Situ Tracing and Capturing of User Feedback on Mobile Phones. Proceedings of the International Conference on Mobile Systems, Applications and Services (Mobisys 2007), $57-70$.

http://doi.org/c8smx6

20. Jon E. Froehlich, Mike Y. Chen, Ian E. Smith, and Fred Potter. (2006). Voting with your Feet: An Investigative Study of the Relationship Between Place Visit Behavior and Preference. Proceedings of the International Conference on Ubiquitous Computing (UbiComp 2006), 333-350.

http://doi.org/dw2bxj

21. Eric Granholm, Catherine Loh, and Joel Swendsen. (2008). Feasibility and Validity of Computerized Ecological Momentary Assessment in Schizophrenia. Schizophrenia Bulletin, 34(3), 507-14. http://doi.org/bb5ch2

22. Eric Hoddes, Vincent Zarcone, and William Dement. (1972). Development and Use of Stanford Sleepiness Scale. Psychophysiology, 9(1), 150.

23. Stefan E Hormuth. (1986). The Sampling of Experiences In Situ. Journal of Personality, 54(1), 262-293.

http://doi.org/fr7bs4

24. James M. Hudson, Jim Christensen, Wendy A. Kellogg, and Thomas Erickson. (2002). "I'd be overwhelmed, but it's just one more thing to do": Availability and Interruption in Research Management. Proceedings of the SIGCHI Conference on Human Factors in Computing Systems (CHI 2002), 97-104. http://doi.org/dq59k7

25. Stephen S. Intille, Emmanuel Munguia Tapia, John Rondoni, Jennifer Beaudin, Chuck Kukla, Sitij Agarwal, Ling Bao, and Kent Larson. (2003). Tools for Studying Behavior and Technology in Natural Settings. Proceedings of the International Conference on Ubiquitous Computing (UbiComp 2003), 157-174. http://doi.org/dghjhz
26. Ashish Kapoor and Eric Horvitz. (2008). Experience Sampling for Building Predictive User Models: A Comparative Study. Proceeding of the SIGCHI Conference on Human Factors in Computing Systems (CHI 2008), 657-666. http://doi.org/d6sr59

27. Predrag Klasnja, Beverly L. Harrison, Louis LeGrand, Anthony LaMarca, Jon E. Froehlich, and Scott E. Hudson. (2008). Using Wearable Sensors and Real Time Inference to Understand Human Recall of Routine Activities. Proceedings of the International Conference on Ubiquitous Computing (UbiComp 2008), 154-163. http://doi.org/fwkjv6

28. Predrag Klasnja and Wanda Pratt. (2012). Healthcare in the Pocket: Mapping the Space of Mobile-Phone Health Interventions. Journal of Biomedical Informatics, 45(1), 184-198.

http://doi.org/c8t7t9

29. William J. Korotitsch and Rosemery O. Nelson-Gray. (1999). An Overview of Self-Monitoring Research in Assessment and Treatment. Psychological Assessment, 11(4), 415-425.

http://doi.org/fs8szw

30. Neal Lathia, Kiran K. Rachuri, Cecilia Mascolo, and Peter J. Rentfrow. (2013). Contextual Dissonance: Design Bias in Sensor-Based Experience Sampling Methods. Proceedings of the ACM International Joint Conference on Pervasive and Ubiquitous Computing (UbiComp 2013), ACM Press, 183-192. http://doi.org/bbpg

31. C.W. Lejuez, Derek R. Hopko, Ron Acierno, Stacey B. Daughters, and Sherry L. Pagoto. (2011). Ten Year Revision of the Brief Behavioral Activation Treatment for Depression: Revised Treatment Manual. Behavior Modification, 35(2), 111-161. http://doi.org/d2cgds

32. Ian Li, Anind K. Dey, and Jodi Forlizzi. (2010). A Stage-Based Model of Personal Informatics Systems. Proceedings of the ACM Conference on Human Factors in Computing Systems (CHI 2010)., 557-566. http://doi.org/bh8zsb

33. C.R. Martell, S. Dimidijian, and R. Herman-Dunn. (2013). Behavioral Activation for Depression: A Clinician's Guide. Guilford Press.

34. Leysia Palen and Marilyn Salzman. (2002). Voice-Mail Diary Studies for Naturalistic Data Capture Under Mobile Conditions. Proceedings of the ACM Conference on Computer Supported Cooperative Work (CSCW 2002), 87-95.

http://doi.org/drg82q 
35. Martin Pielot, Tilman Dingler, Jose San Pedro, and Nuria Oliver. (2015). When Attention is Not Scarce Detecting Boredom from Mobile Phone Usage. Proceedings of the ACM International Joint Conference on Pervasive and Ubiquitous Computing (UbiComp 2015), 825-836. http://doi.acm.org/10.1145/2750858.2804252

36. John Rieman. (1993). The Diary Study: A WorkplaceOriented Research Tool to Guide Laboratory Efforts. Proceedings of the SIGCHI Conference on Human Factors in Computing Systems (CHI 1993), 321-326. http://doi.org/cxn7sw

37. John Rooksby, Mattias Rost, Alistair Morrison, and Matthew Chalmers Chalmers. (2014). Personal Tracking as Lived Informatics. Proceedings of the ACM Conference on Human Factors in Computing Systems (CHI 2014), 1163-1172. http://doi.org/bbdz

38. Stephanie Rosenthal, Anind K. Dey, and Manuela Veloso. (2011). Using Decision-Theoretic Experience Sampling to Build Personalized Mobile Phone Interruption Models. Proceedings of the International Conference on Pervasive Computing (Pervasive 2011), 170-187. http://doi.org/d2fcbj

39. James A. Russell, Anna Weiss, and Gerald A. Mendelsohn. (1989). Affect Grid: A Single-Item Scale of Pleasure and Arousal. Journal of Personality and Social Psychology, 57(3), 493-502.

40. Saul Shiffman. (2009). Ecological Momentary Assessment (EMA) in Studies of Substance Use. Psychological Assessment, 21(4), 486-497. http://doi.org/csttmw

41. Timothy Sohn, Kevin A. Li, William G. Griswold, and James D. Hollan. (2008). A Diary Study of Mobile Information Needs. Proceeding of the CHI Conference on Human Factors in Computing Systems (CHI 2008), 433-442.

http://doi.org/fjrb85
42. Arthur A. Stone, Christine A. Bachrach, Jared B. Jobe, Howard S. Kurtzman, and Virginia S. Cain (eds.). (1999). The Science of Self-Report: Implications for Research and Practice. Psychology Press.

43. Arthur A. Stone, Saul Shiffman, Joseph E Schwartz, Joan E Broderick, and Michael R Hufford. (2003). Patient Compliance with Paper and Electronic Diaries. Controlled Clinical Trials, 24(2), 182-199.

http://doi.org/bnwr3b

44. Arthur A. Stone and Saul Shiffman. (2003). Ecological Momentary Assessment (EMA) in Behavorial Medicine. Journal of Happiness Studies, 4(1), 35-52. http://doi.org/bdjp7x

45. Edison Thomaz, Irfan Essa, and Gregory D. Abowd. (2015). A Practical Approach for Recognizing Eating Moments with Wrist-Mounted Inertial Sensing. Proceedings of the 2015 ACM International Joint Conference on Pervasive and Ubiquitous Computing (UbiComp 2015), 1029-1040. http://dx.doi.org/10.1145/2750858.2807545

46. Khai N. Truong, Thariq Shihipar, and Daniel J. Wigdor. (2014). Slide to X: Unlocking the Potential of Smartphone Unlocking. Proceedings of the ACM Conference on Human Factors in Computing Systems (CHI 2014), 3635-3644.

http://doi.org/bbph

47. Rajan Vaish, Keith Wyngarden, Jingshu Chen, Brandon Cheung, and Michael S. Bernstein. (2014). Twitch Crowdsourcing: Crowd Contributions in Short Bursts of Time. Proceedings of the ACM Conference on Human Factors in Computing Systems (CHI 2014), 3645-3654.

http://doi.org/bbpj 